\title{
Increased expression of neutrophil gelatinase-associated lipocalin receptor by interleukin-1 $\beta$ in human mesangial cells via MAPK/ERK activation
}

\author{
SHANSHAN MAO, TAO JIANG, GUOGUO SHANG, ZHENGSHENG WU and NONG ZHANG \\ Department of Pathology, Shanghai Medical College, Fudan University, Shanghai, P.R. China
}

Received November 19, 2010; Accepted January 14, 2011

DOI: $10.3892 / \mathrm{ijmm} .2011 .613$

\begin{abstract}
Neutrophil gelatinase-associated lipocalin (NGAL), one of the most promising next-generation biomarkers in clinical nephrology, has received extensive attention. However, the basic role of its receptor (NGALR) remains unclear. Here, we have assessed the expression pattern of NGALR in injured glomeruli and explored the possible mechanism of the NGALR involvement in inflammation in human mesangial cells (HMC). The expression pattern of NGALR was detected by immunohistochemistry in biopsy samples of 93 glomerulonephritis patients and healthy controls, and the regulation of NGALR by the proinflammatory cytokines, TGF- $\beta 1$, TNF- $\alpha$ and IL-1 $\beta$ in HMC was analyzed by real-time PCR and Western blotting. NGALR was found to be expressed in glomeruli. Its expression was significantly higher in acute proliferative glomerulonephritis and lupus nephritis than that in other types of glomerulonephritis or healthy kidney tissues. In in vitro experiments, both mRNA and protein levels of NGALR were dramatically induced by treatment of IL-1 $\beta$, whereas TGF- $\beta 1$ or TNF- $\alpha$ did not have the same effect. Furthermore, it was shown that the IL- $1 \beta$-induced NGALR expression is mediated via the MAPK/ERK signaling pathway by using pharmacological inhibitors. Interestingly, the basal mRNA levels of NGAL detected in HMC, could be induced by IL-1 $\beta$. However, NGAL protein could not be detected, even with IL-1 $\beta$ treatment. The ability of HMC to express NGAL protein was ascertained by exogenous administration of NGAL. In conclusion, the data show that NGALR is differentially expressed in human glomerular disease and is significantly up-regulated by Il-1 $\beta$ in HMC via MAPK/ERK activation. Furthermore, exogenous NGAL can be uptaken into HMC.
\end{abstract}

Correspondence to: Professor Nong Zhang, Department of Pathology, Shanghai Medical College, Fudan University, 138 Yixueyuan Road, Shanghai 200032, P.R. China

E-mail: nzhang@fudan.edu.cn

Key words: neutrophil gelatinase-associated lipocalin receptor, mesangial cells, glomerulonephritis, IL-1 $\beta$

\section{Introduction}

Neutrophil gelatinase-associated lipocalin (NGAL) is a $25-\mathrm{kDa}$ protein which belongs to the lipocalin superfamily. NGAL was initially detected in activated neutrophils (1), in accordance with its role as an innate antibacterial factor (2). NGAL receptor (NGALR) is one of the cellular receptors of NGAL identified recently (3), which was first isolated from murine FL5.12 cells (4). This specific receptor probably has a fundamental role in NGAL endocytosis and cellular trafficking $(4,5)$.

NGAL protein accumulation in the blood and urine can be detected only within a few hours of acute kidney injury (AKI) (6-9). These characteristics of NGAL have made it a promising biomarker of AKI, abundant in the blood and urine (5,10-14). Furthermore, recent evidence also suggests that NGAL may be involved in the pathophysiological process of chronic kidney diseases (CKD), such as polycystic kidney disease and chronic glomerulonephritis (15). Patients with systemic lupus erythematosus, IgA nephropathy, membranous (MGN) or membranoproliferative glomerulonephritis (MPGN) have higher urinary and serum NGAL levels compared to normal controls (16-19). However, the function of NGAL besides being a biomarker, remains unclear. Further research is needed to ascertain the role of this protein in the pathophysiological processes of some CKD-associated conditions.

Several studies have investigated the relationship between NGALR and diseases. Cui et al (20) have shown that NGALR hypomethylation contributes to its expression in esophageal carcinomas and that this overexpression may play a role in the pathogenesis of this disease. Vinuesa et al (21) have presented evidence indicating that the regenerative function of $\operatorname{Lcn} 2$ (the murine homolog of NGAL) is influenced by the inflammatory status of the kidney and the expression of Lcn2 receptors. The expression of NGALR in the kidney, especially in the injured glomeruli is unclear, and furthermore, no studies have demonstrated the effects of pro-inflammatory cytokines on NGALR expression.

Although it is believed that there is nearly no expression of NGAL in glomeruli (22), where intrinsic cells, such as the mesangial cells, play important roles in the occurrence and development of renal fibrosis (23), it is possible that some intrinsic cells in glomeruli could express NGALR, the specific 
receptor of NGAL. It is thus possible, that NGAL could have some function in chronic glomerulonephritis via being recruited by NGALR and engulfed by intrinsic cells.

Therefore, the present study focused on the expression of NGALR in several types of glomerulonephritis by using immunohistochemistry. We also evaluated the expression of NGALR in mesangial cells under inflammatory conditions and the corresponding alterations in NGAL levels. Our research should provide useful data for investigation of the possible roles of NGAL-NGALR in the progression of CKD.

\section{Materials and methods}

Patients. In order to examine the expression of NGALR in the human injured glomeruli, renal biopsy samples were used for immunohistochemical staining. Ninety-three cases of kidney needle biopsies, including 6 cases of healthy kidney tissue (distant from the kidney tumour), 14 cases of MPGN, 10 cases of minimal change disease (MCD), 9 cases of focal segmental glomerulosclerosis (FSGS), 18 cases of $\operatorname{Ig}$ A nephropathy, 11 cases of MGN, 6 cases of acute proliferative glomerulonephritis (APGN) and 19 cases of lupus nephritis (LN), were obtained from the Department of Pathology, Shanghai Medical College, Fudan University, in accordance with local ethics guidelines. All the patients were diagnosed by light microscopy, electron microscopy and immunofluorescence.

Immunohistochemistry. Briefly, deparaffinized $2 \mu \mathrm{m}$ sections were stained with rabbit anti-NGALR antibody (ProSci Inc., USA). The concentration of the antibody was $1.5 \mu \mathrm{g} / \mathrm{ml}$. Immobilized antibodies were detected by the avidin-biotinperoxidase technique (Vector Laboratories, UK). DAB was used as the chromogen and hematoxylin as the nuclear counterstaining agent. The primary antibody was omitted in the negative control. The positive case rate of staining was defined as the proportion of cases with positive staining among the total number of cases in each group (24).

Cell culture. HMC were purchased from (ScienCell Research Laboratories, USA). HMC were incubated in RPMI-1640 with $10 \%$ newborn serum at $37^{\circ} \mathrm{C}$ in $95 \%$ air and $5 \% \mathrm{CO}_{2}$ at a concentration of $2 \times 10^{5}$ cells $/ 2 \mathrm{ml}$ in a 6 -well tissue culture plate. After $24 \mathrm{~h}$ the HMC were treated with $10 \mathrm{ng} / \mathrm{ml}$ TGF- $\beta 1$ (R\&D Systems, USA), $10 \mathrm{ng} / \mathrm{ml}$ TNF- $\alpha$ (Sigma, USA) or $10 \mathrm{ng} / \mathrm{ml} \mathrm{IL-1 \beta}$ (Sigma, USA), respectively for 12, 24 and $48 \mathrm{~h}$ or with $10 \mu \mathrm{M}$ UO126 (Cell Signaling Technologies, USA). The concentrations of these cytokines were selected according to previous published studies $(25,26)$.

Total RNA isolation and RT-PCR. Total RNA was extracted from cells with TRIzol (Invitrogen, USA). First-strand cDNA was reverse transcribed from $2 \mu \mathrm{g}$ total RNA in a total volume of $20 \mu \mathrm{l}$ using the Reverse Transcription System (Takara, Japan) according to the manufacturer's instructions.

Semiquantitative PCR was performed using Taq DNA polymerase (Takara) in a Bio-Rad S1000 Thermal Cycler. After initial denaturation $\left(5 \mathrm{~min}\right.$ at $94^{\circ} \mathrm{C}$ ), cDNA was subjected to 30 cycles of PCR. The primer set for the human NGAL was forward 5'-TCACCTCCGTCCTGTTTAGG-3' and reverse 5'-CGAAGTCAGCTCCTTGGTTC-3' (27). The expression of $\beta$-actin was used for normalization. The $\beta$-actin primer set was forward 5'-TTCTACAATGAGCTGCGTGTGG-3' and reverse 5'-GTGTTGAAGGTCTCAAACATGAT-3'. The PCR annealing temperature was $60^{\circ} \mathrm{C}$ for human NGAL and $59^{\circ} \mathrm{C}$ for $\beta$-actin. PCR products were separated on a $2 \%$ agarose gel.

Real-time PCR. The oligonucleotide primers designed for NGALR1, NGALR2, and $\beta$-actin were as follows: NGALR1, 5'-TGGGATTGTGAGCATCCTATCTT-3' and 5'-GGCAGC CTCGTTCAGATCTC-3'; NGALR2, 5'-GGCAAAATCGAC AACACTGTAGAG-3' and 5'-CGTGGGCACCGCATCT-3'; $\beta$-actin, 5'-CAACTGGGACGACATGGAGAAA-3' and 5'-GA TAGCAACGTACATGGCTGGG-3' (20). Each primer spanned a different exon to avoid amplification of contaminating genomic DNA.

Real-time quantitative PCR was performed using the Rotor-Gene 3000 (Corbett Research, Australia). Briefly, each PCR mixture contained cDNA, Premix ExTaq (Takara) and a primer pair in a final volume of $20 \mu \mathrm{l}$. After activation of Taq polymerase at $95^{\circ} \mathrm{C}$ for $10 \mathrm{sec}$, PCR was performed for $40-50$ cycles, with each cycle consisting of a denaturion step at $95^{\circ} \mathrm{C}$ for $5 \mathrm{sec}$, annealing, and extending at $60^{\circ} \mathrm{C}$ for $30 \mathrm{sec}$. These served as the standard curves from which we determined the rate of change in the threshold cycle values. The amount of target gene expression was calculated from the standard curve, and quantitative normalization of NGALR1 and NGALR 2 cDNA in each sample was performed using $\beta$-actin as an internal control.

Western blot analysis. Cells were lysed quickly using 1X SDS buffer on ice, boiled for $5 \mathrm{~min}$ and then stored at $-80^{\circ} \mathrm{C}$ until use. Samples were subjected to $10 \%$ SDS-PAGE, transferred to polyvinylidene difluoride membranes (Millipore, Eschborn, Germany). The membranes were probed with antibodies against NGALR (ProSci Inc.), NGAL (Abcam, USA), phosphop44/42MAPK (Cell Signaling Technologies), p44/42MAPK (Cell Signaling Technologies) and $\beta$-actin (Sigma) as a loading control for $1 \mathrm{~h}$ at $37^{\circ} \mathrm{C}$, then incubated overnight at $4^{\circ} \mathrm{C}$. Following incubation with HRP-conjugated secondary antibodies (Cell Signaling Technologies), the immunoreaction was visualized by enhanced chemiluminescence and film exposure. Each experiment was repeated at least three times.

Statistical analysis. Statistics were calculated using SPSS software. Statistical comparisons between groups were made using the Fisher's exact test and the analysis of variance (ANOVA) with pairwise multiple comparisons made using the Fisher's protected least significant difference test. A p-value of $<0.05$ was defined as statistically significant.

\section{Results}

Expression of NGALR in the glomeruli from different types of glomerulonephritis. Using immunohistochemistry, the positive signal of NGALR expression was observed in glomeruli of APGN and LN, while in IgA nephropathy, MCD, MPGN, MGN, FSGS and healthy kidney tissue, the signals were almost negative. The positive staining was mainly distributed within the mesangium with low expression in the peripheral 
Table I. The positive case rate of NGALR expression within the glomeruli of some types of glomerulonephritis.

\begin{tabular}{lccc}
\hline & Number of cases & Number of positive cases & Positive case rate (\%) \\
\hline Acute diffuse proliferative GN & 6 & 6 & $100^{\mathrm{a}}$ \\
Lupus nephritis & 19 & 17 & $89.47^{\mathrm{a}}$ \\
Minimal change disease & 10 & 1 & 10.00 \\
FSGS & 9 & 1 & 11.11 \\
IgA nephropathy & 18 & 3 & 16.67 \\
Membranous GN & 11 & 1 & 9.09 \\
Membranoproliferative GN & 14 & 1 & 7.14 \\
Healthy kidney tissue & 6 & 1 & 16.67
\end{tabular}

Overall, 93 cases were enrolled. The positive case rate increased significantly in the first two groups. ${ }^{\text {a }}<<0.01$ compared to healthy controls; GN, glomerulonephritis; FSGS, focal segmental glomerulosclerosis.
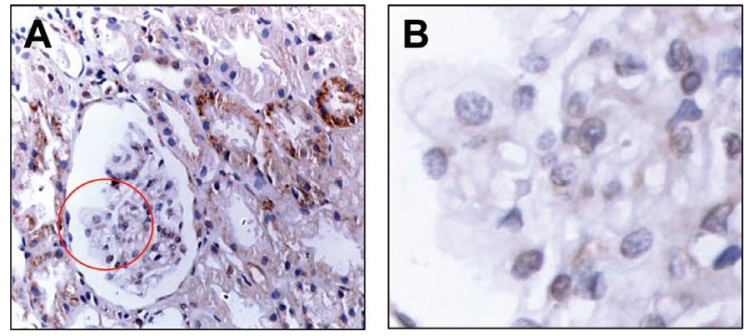

MCD
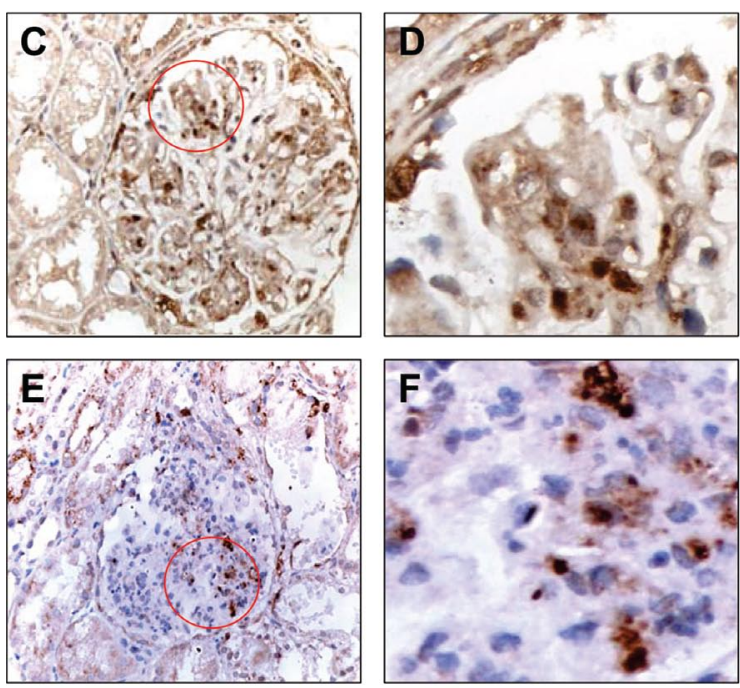

IHC-NGALR

Figure 1. NGALR expression in the glomeruli of different types of glomerulonephritis (GN). NGALR was not detected in the glomeruli of minimal change disease (A and B). In lupus nephritis ( $\mathrm{C}$ and $\mathrm{D})$ and acute proliferative $\mathrm{GN}$ ( $\mathrm{E}$ and $\mathrm{F})$, the positive staining was mainly distributed at the mesangium with a little expression in the the peripheral area of the capillary tufts. In addition, the parietal epithelia of the Bowman's capsule and distal tubular epithelial cells were partially positive. (B, D and F) high-power detail view of the circles in (A, C and E). Hematoxylin was used for the nuclear counterstaining. MCD, minimal change disease; LN, lupus nephritis; APGN, acute proliferative glomerulonephritis.

area of the capillary tufts. Positive staining was also observed in some parietal epithelia of the Bowman's capsule, crescents and a portion of proximal tubular epithelial cells in all the cases (Fig. 1). In addition, the degree of NGALR expression in the glomeruli was somewhat different among the various types of glomerulonephritis. The proportion of positive cases was $100 \%$ in APGN, $89 \%$ in LN, while it was only about $10 \%$ in other types of glomerulonephritis, nearly similar to that in healthy kidney tissue (Table I). The statistical analysis demonstrated that the positive case rate was significantly higher in LN and APGN than in IgA nephropathy, MGN, MCD, FSGS, MPGN and healthy kidney tissues $(\mathrm{p}<0.01)$. There were no statistically significant differences between FSGS, MCD, minor abnormality and healthy kidney tissue $(\mathrm{p}>0.05)$.

Elevated expression of NGALR in HMC mediated by $I L-1 \beta$ in vitro. In order to screen for possible inducers of NGALR expression in some types of nephrites, normal HMC were treated by the common pro-inflammatory cytokines, TGF- $\beta 1$ $(10 \mathrm{ng} / \mathrm{ml})$, TNF- $\alpha(10 \mathrm{ng} / \mathrm{ml})$ and IL-1 $\beta(10 \mathrm{ng} / \mathrm{ml})$ for 12,24 and $48 \mathrm{~h}$. Western blot analysis and real-time PCR revealed that the basal protein levels of NGALR were low in normal HMCs (Fig. 2A) and somewhat increased in the groups exposed to the cytokines, TGF- $\beta 1$ and TNF- $\alpha$, with no significant differences compared to the normal control (Fig. 2C and D).

The protein levels of NGALR, were up-regulated from their basal levels by IL- $1 \beta$ treatment as determined by Western blot analysis. The increased exprssion was time- but not dose-dependent, and the maximal induction was observed $48 \mathrm{~h}$ after treatment and was 4-fold higher (Fig. 2B and E).

As determined by real-time PCR, the mRNA expression of the alternatively spliced NGALR variants, NGALR1 and NGALR2 (20), were significantly increased in the groups exposed to IL-1 $\beta$ treatment compared to the normal controls, and this increased expression was time-dependent (Fig. 3).

The IL-1 $\beta$-induced increase in the expression of NGALR is mediated through activation of the MAPK/ERK signaling pathway. We further investigated which molecules are implicated in IL-1 $\beta$ signaling, which in turn may mediate the positive effect of IL-1 $\beta$ on NGALR expression. The ability of IL- $1 \beta$ to activate MAPK has been examined in mesangial cells (28). We demonstrated that the increased NGALR expression induced by IL-1 $\beta$ requires the activation of the 
A

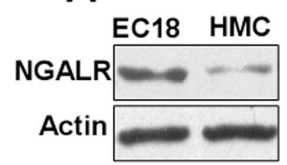

C

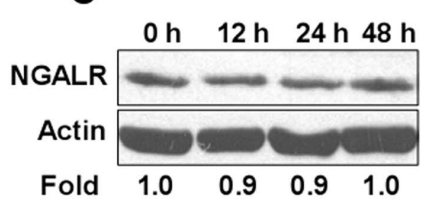

B

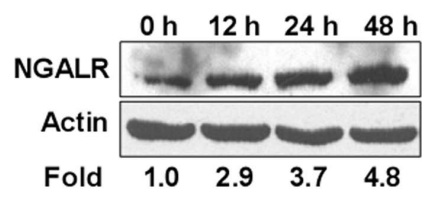

D

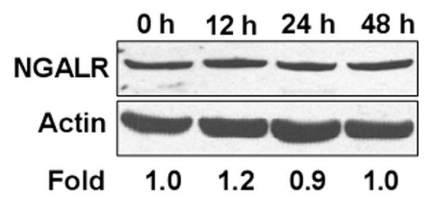

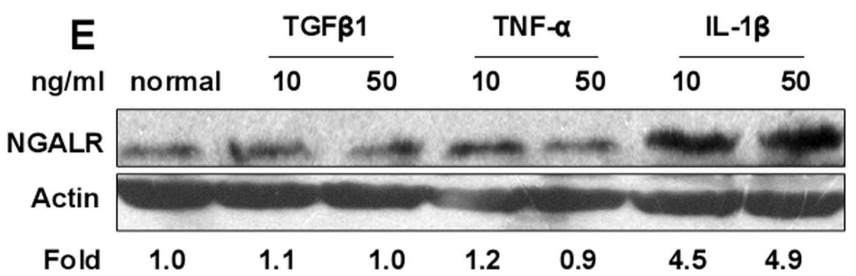

Figure 2. Induction of NGALR protein levels by IL-1 $\beta$ in HMC. Western blot analysis demonstrated the basal level of NGALR protein expression in normal HMC (A) and a small rise in the groups that were treated with TGF- $\beta 1$ (C) or TNF- $\alpha$ (D) for different times. An obvious increase in NGALR protein levels in the groups treated with IL-1 $\beta$ (B) was found and this increase was concentration-independent $(\mathrm{E})$. The left band in A is the positive control of esophageal cancer cell (EC18). The fold-induction relative to the control was determined. Data are the mean $\pm \mathrm{SD}$ of values from three independent experiments.
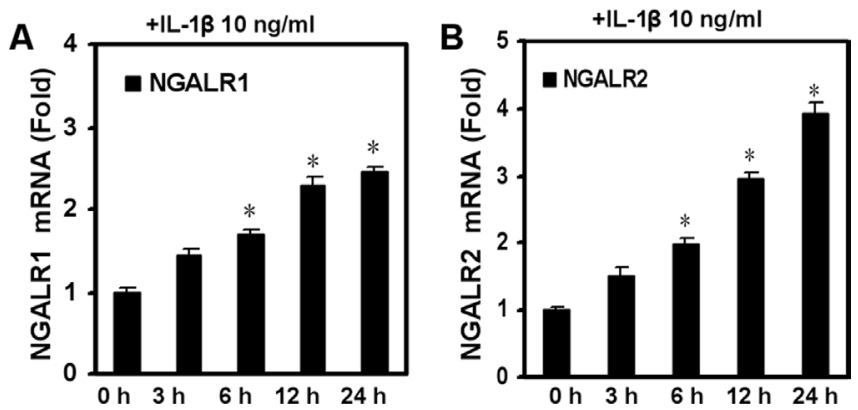

Figure 3. Induction of NGALR mRNA by IL-1 $\beta$ in HMC. Expression of NGALR1 (A) and NGALR2 (B) mRNA increased in HMC cultured with IL-1 $\beta$ for different times. The representative results demonstrate the timedependent up-regulation of NGALR mRNA expression by IL-1 $\beta$. Relative levels of individual mRNAs were normalized to those of $\beta$-actin mRNA. Data are the mean \pm SD of values from three independent experiments.

MAPK/ERK pathway. IL-1 $\beta$ stimulation resulted in a rapid, transient phosphorylation of ERK (Fig. 4A). ERK activation was maximal at $3 \mathrm{~h}$ (at which point it was about 4 -fold higher) and returned to basal levels after $6 \mathrm{~h}$. Next, in order to investigate whether ERK activation is a key point in the induction of NGALR expression by IL-1 $\beta$, we examined the effects of a MAPK/ERK inhibitor on the IL-1 $\beta$-induction of NGALR. HMC were treated for $1 \mathrm{~h}$ with the ERK inhibitor, U0126 $(10 \mu \mathrm{M})$ followed by stimulation with $10 \mathrm{ng} / \mathrm{ml}$ IL-1 $\beta$ for $16 \mathrm{~h}$. The increased expression of NGALR was partly inhibited following treatment with U0126 (Fig. 4B). In addition, when HMC were treated for $1 \mathrm{~h}$ with the U0126

A

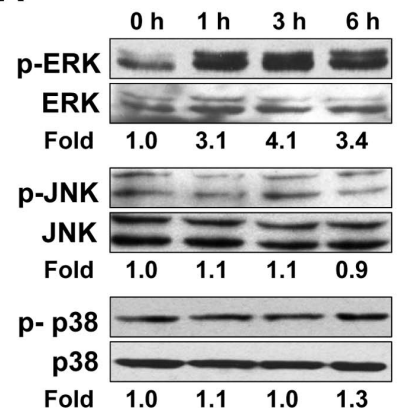

B
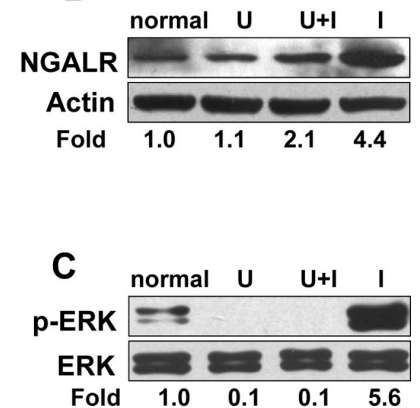

Figure 4. Roles of MAPKs in IL-1 $\beta$-mediated NGALR induction. (A) Whole cell lysates from HMC stimulated for the indicated number of hours with $10 \mathrm{ng} / \mathrm{ml} \mathrm{IL-1} \beta$ were separated by SDS-PAGE and immunoblotted with an antibody to phosphorylated ERK, JNK, p38 followed by immunoblotting with an antibody to total ERK, JNK, p38 to determine equality of loading. (B) Cells were preincubated for $1 \mathrm{~h}$ in RPMI-1640 with or without $10 \mu \mathrm{M}$ U0126 (U) followed by Western blotting with or without $10 \mathrm{ng} / \mathrm{ml} \mathrm{IL-1 \beta}$ (I) for $16 \mathrm{~h}$. Total cell lysates were then subjected to Western blot analysis with antibodies against NGALR and $\beta$-actin. (C) Cells were preincubated for $1 \mathrm{~h}$ with or without $10 \mu \mathrm{M}$ U0126 following stimulation with $100 \mathrm{ng} / \mathrm{ml} \mathrm{IL-1 \beta}$ for $3 \mathrm{~h}$, and then total cell lysates were subjected to Western blot analysis with antibodies to phospho-ERK and ERK. The results show that IL-1 $\beta$ induced NGALR expression was partly ERK-dependent. The fold-induction relative to the control was determined. Data are the mean \pm SD of values from three independent experiments.
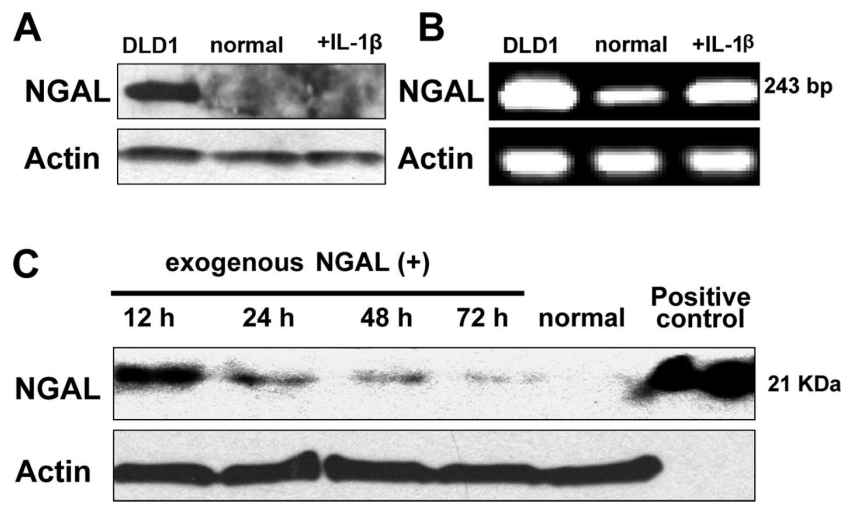

Figure 5. Expression of NGAL in HMC. (A) No expression of NGAL protein in HMC with or without IL-1 $\beta$ treatment. (B) NGAL mRNA was expressed in HMC and the expression increased with IL- $1 \beta$ treatment. The left band in (A) and (B) is the positive control of the colon cancer cell line, DLD1. (C) The protein expression of NGAL in HMC cultured with exogenous NGAL $(50 \mathrm{ng} / \mathrm{ml})$ for the indicated times. The last band is the positive control of exogenous NGAL. Data were obtained from three separate experiments.

following stimulation with $10 \mathrm{ng} / \mathrm{ml} \mathrm{IL}-1 \beta$ for $3 \mathrm{~h}$, the ERK inhibitor U0126 strongly suppressed the phosphorylation of ERK, which indicated that it specifically inhibits ERK activity (Fig. 4C). These results suggest that ERK is one of the signaling pathways contributing to the IL-1 $\beta$-mediated NGALR induction in HMC.

Changes of NGAL expression in HMC. First, we confirmed by PCR and Western blot analysis that NGAL mRNA was expressed in HMC, even though no NGAL protein could be detected. Furthermore, NGAL mRNA was induced by IL-1 $\beta$ (Fig. 5A and B). To further investigate whether HMCs could engulf NGAL that was bound to NGALR, we analyzed the 
NGAL protein level in HMC culture medium at different time-points after adding exogenous NGAL. The results demonstrate a time-dependent decrease of NGAL protein levels in cultured $\mathrm{HMC}$, which indicates the uptake of NGAL by HMC (Fig. 5C). This phenomenon is in accordance with the in vivo protein degradation process.

\section{Discussion}

NGAL is a $25-\mathrm{kDa}$ glycoprotein, first found to be an acute phase reactant secreted by the liver and then identified as a matrix protein of specific granules of human neutrophils (29). During inflammation or exposure to serum, LPS, and cytokines such as the human fibroblast growth factor and IL-1 $\beta$ (30-33), NGAL has been found to be strongly induced in several organs and in a variety of cultured cells, such as the renal tubular epithelial cells.

Since it can be induced within a few hours in the blood and urine during AKI, NGAL levels may predict the acute worsening of unstable nephropathies and even the future appearance of AKI after treatments. Furthermore, recent evidence suggests that NGAL may be involved in the pathophysiological process of CKD, such as polycystic kidney disease and glomerulonephritis (15). In these renal diseases, NGAL levels in the blood and urine are altered.

It is generally acknowledged that in addition to NGAL being the most promising next-generation biomarker in clinical nephrology, it promotes renal tubular epithelial cell growth and differentiation in response to ischemic injuries (32). Currently, despite some reasearch in progress, the biological significance of NGAL in CKD, especially in glomerulonephritis needs further examination.

Recently, the cellular surface receptor, NGALR, has been identified as one of the NGAL specific receptors (3), which probably has a fundamental role in NGAL endocytosis and cellular trafficking. A series of studies has suggested that NGAL is internalized inside the cell as a protein alone (Apo-NGAL) or in a complex with iron-binding siderophores (Holo-NGAL) after interaction with NGALR, which mediates a new iron delivery pathway that functions distinctly from the transferrin pathway $(5,34)$. However, the expression of NGALR or the effect of pro-inflammatory cytokines on NGALR expression in the kidneys is not well known especially in renal glomeruli of various types of glomerulonephritis.

It is generally known that HMC play a crucial role in glomeruli under both normal physiological and pathological conditions. They provide structural support and regulate the glomerular filtration rate (35). In addition, the mesangium serves as a source and target of various kinds of cytokines, thus contributing to the pathophysiology of various types of glomerular diseases (36). Aberrant proliferation of mesangial cells and extracellular matrix deposition is a common finding in several types of glomerulonephritis induced by a variety of initial insults, whether metabolic (as in diabetic nephropathy) or immunological (as in IgA nephropathy and LN) (23).

Therefore, it is important to detect the expression of NGALR in mesangial cells in order to study the biological significance of NGAL in chronic renal diseases. The hypothesis is that NGAL could play a role in CKD, via interaction with the NGALR which was found in intrinsic cells within glomeruli.
We postulate that NGALR is an important factor involved in the pathological response to decreased NGAL expression in an in vivo microenvironment.

The present study shows that the expression of NGALR protein was significantly higher in renal glomeruli of APGN and LN compared to normal kidney tissue and other types of glomerulonephritis, and that the positive staining was mainly distributed at the mesangium. However, whether NGALR is expressed and secreted by HMC or some other cell types remains uncertain. We therefore, performed further in vivo experiments. The results illustrate that NGALR protein is expressed in HMC.

Further examination is needed to define the reasons why NGALR protein levels were higher in APGN and LN than in the other types of glomerulonephritis. It is worth noting that inflammation is a common feature in glomerulonephritis and worsens during the progression toward end-stage renal disease (ESRD). We must point out the existence of severe inflammatory cells and the occurence of neutrophilic granulocyte infiltration in APGN and LN (especially in class IV). In order to investigate the possible reasons, we chose to use common pro-inflammatory cytokines to stimulate HMC. These data were confirmed in in vitro cell experiments by RT-PCR and Western blot analyses. We found that NGALR mRNA and protein levels were up-regulated in HMC stimulated by IL-1 $\beta$, but not by TGF- $\beta 1$ or TNF- $\alpha$, and that this up-regulation was time- and dose-independent. Furthermore, our results demonstrated that IL-1 $\beta$-induced NGALR expression was mediated via the MAPK/ERK signaling pathway. The data indicate that the up-regulation of NGALR might be associated with IL-1 $\beta$ induced inflammatory gene expression in HMC.

Immunohistochemistry results demonstrated that NGAL was highly expressed in proximal tubular epithelial cells in the normal and injured kidney, with low expression in other areas of the kidney, such as the mesangium (22). We also found that NGAL protein was absent from normal or stimulated HMC, whereas NGAL mRNA was up-regulated in HMC treated with IL-1 $\beta$ and this increasing expression was time-dependent. This illustrates that the process of protein translation is blocked in vivo. However, the protein expression of NGAL in HMCs was detected upon exogenous treatment with NGAL. Whether NGAL in the in vivo microenvironment could be delivered into mesangial cells by NGALR, needs further experimentation. However, our research provides fundamental data for the possible roles of NGAL-NGALR in the progression of CKD. Future studies are needed to further address this issue.

\section{Acknowledgements}

We are grateful to Dr JianHua Li for useful discussions. This study was supported by a grant from the National Natural Science Foundation of China (NSFC 30570857).

\section{References}

1. Kjeldsen $\mathrm{L}$, Johnsen $\mathrm{AH}$, Sengelov $\mathrm{H}$, et al: Isolation and primary structure of NGAL, a novel protein associated with human neutrophil gelatinase. J Biol Chem 268: 10425-10432, 1993.

2. Goetz DH, Holmes MA, Borregaard N, et al: The neutrophil lipocalin NGAL is a bacteriostatic agent that interferes with siderophore-mediated iron acquisition. Mol Cell 10: 1033-1043, 2002. 
3. Fang WK, Xu LY, Lu XF, et al: A novel alternative spliced variant of neutrophil gelatinase-associated lipocalin receptor in oesophageal carcinoma cells. Biochem J 403: 297-303, 2007.

4. Devireddy LR, Gazin C, Zhu X, et al: A cell surface receptor for lipocalin $24 \mathrm{p} 3$ selectively mediates apoptosis and iron uptake. Cell 123: 1293-1305, 2005.

5. Schmidt-Ott KM, Mori K, Li JY, et al: Dual action of neutrophil gelatinase-associated lipocalin. J Am Soc Nephrol 18: 407-413, 2007.

6. Mishra J, Dent C, Tarabishi R, et al: Neutrophil gelatinaseassociated lipocalin (NGAL) as a biomarker for acute renal injury after cardiac surgery. Lancet 365: 1231-1238, 2005.

7. Mishra J, Ma Q, Prada A, et al: Identification of neutrophil gelatinase associated lipocalin as a novel early urinary biomarker for ischemic renal injury. J Am Soc Nephrol 14: 2534-2543, 2003.

8. Wagener G, Jan M, Kim M, et al: Association between increases in urinary neutrophil gelatinase-associated lipocalin and acute renal dysfunction after adult cardiac surgery. Anesthesiology 105: 485-491, 2006.

9. Bennett M, Dent CL, Ma Q, et al: Urine NGAL predicts severity of acute kidney injury after cardiac surgery: a prospective study. Clin J Am Soc Nephrol 3: 665-673, 2008.

10. Mori K and Nakao K: Neutrophil gelatinase-associated lipocalin as the realtime indicator of active kidney damage. Kidney Int 71 967-970, 2007.

11. Devarajan P: Emerging biomarkers of acute kidney injury. Contrib Nephrol 156: 203-212, 2007.

12. Coca SG, Yalavarthy R, Concato J, et al: Biomarkers for the diagnosis and risk stratification of acute kidney injury: a systematic review. Kidney Int 73: 1008-1016, 2008.

13. Waikar SS and Bonventre JV: Biomarkers for the diagnosis of acute kidney injury. Curr Opin Nephrol Hypertens 16: 557-564, 2007.

14. Nickolas TL, O'Rourke MJ, Yang J, et al: Sensitivity and specificity of a single emergency department measurement of urinary neutrophil gelatinase-associated lipocalin for diagnosing acute kidney injury. Ann Intern Med 148: 810-819, 2008.

15. Bolignano D, Donato V, Coppolino G, et al: Neutrophil gelatinase-associated lipocalin (NGAL) as a marker of kidney damage. Am J Kidney Dis 52: 595-605, 2008.

16. Brunner HI, Mueller M, Rutherford C, et al: Urinary neutrophil gelatinase-associated lipocalin as a biomarker of nephritis in childhood-onset systemic lupus erythematosus. Arthritis Rheum 54: 2577-2584, 2006.

17. Mitsnefes MM, Kathman TS, Mishra J, et al: Serum neutrophil gelatinase-associated lipocalin as a marker of renal function in children with chronic kidney disease. Pediatr Nephrol 22: 101-108, 2007.

18. Ding H, He Y, Li K, et al: Urinary neutrophil gelatinaseassociated lipocalin (NGAL) is an early biomarker for renal tubulointerstitial injury in IgA nephropathy. Clin Immunol 123 : 227-234, 2007.

19. Bolignano D, Coppolino G, Campo S, et al: Urinary neutrophil gelatinase-associated lipocalin (NGAL) is associated with severity of renal disease in proteinuric patients. Nephrol Dial Transplant 23: 414-416, 2008.
20. Cui L, Xu LY, Shen ZY, et al: NGALR is overexpressed and regulated by hypomethylation in esophageal squamous cell carcinomas. Clin Cancer Res 14: 7674-7681, 2008.

21. Vinuesa E, Sola A, Jung M, Alfaro V and Hotter G: Lipocalin-2induced renal regeneration depends on cytokines. Am J Physiol Renal Physiol 295: F1554-F1562, 2008.

22. Friedl A, Stoesz SP, Buckley P, et al: Neutrophil gelatinaseassociated lipocalin in normal and neoplastic human tissues. Cell type-specific pattern of expression. Histochem $\mathbf{J} 31$ : 433-441, 1999.

23. Cove-Smith A and Hendry BM: The regulation of mesangial cell proliferation. Nephron Exp Nephrol 108: e74-e79, 2008.

24. Liu XL, Furuya T, Li DY, et al: Connexin 26 expression correlates with less aggressive phenotype of intestinal typegastric carcinomas. Int J Mol Med 25: 709-716, 2010.

25. Nakayama Y, Kato N, Nakajima Y, et al: Effect of TNFa on human osteosarcoma cell line Saos2-TNF $\alpha$ regulation of bone sialoprotein gene expression in Saos2 osteoblast-like cells. Cell Biol Int 28: 653-660, 2004.

26. Tsukihara S, Harada T, Deura I, et al: Interleukin-1 $\beta$-induced expression of IL- 6 and production of human chorionic gonadotropin in human trophoblast cells via nuclear factor- $\kappa \mathrm{B}$ activation. Am J Reprod Immunol 52: 218-223, 2004.

27. Roudkenar MH, Halabian R, Roushandeh AM, et al: Lipocalin 2 regulation by thermal stresses: protective role of $\mathrm{Lcn} 2 / \mathrm{NGAL}$ against cold and heat stresses. Exp Cell Res 315: 3140-3151, 2009.

28. Sanchez-Lopez E, Rodriguez-Vita J, Cartier C, et al: Inhibitory effect of interleukin-1 $\beta$ on angiotensin II-induced connective tissue growth factor and type IV collagen production in cultured mesangial cells. Am J Physiol Renal Physiol 294: F149-F160, 2008.

29. Kjeldsen L, Bainton DF, Sengeløv H, et al: Identification of neutrophil gelatinase-associated lipocalin as a novel matrix protein of specific granules in human neutrophils. Blood 83: 799-807, 1994.

30. Liu Q and Nilsen-Hamilton M: Identification of a new acute phase protein. J Biol Chem 270: 22565-22570, 1995.

31. Nielsen BS, Borregaard N, Bundgaard JR, et al: Induction of NGAL synthesis in epithelial cells of human colorectal neoplasia and inflammatory bowel diseases. Gut 38: 414-420, 1996.

32. Gwira JA, Wei F, Ishibe S, et al: Expression of neutrophil gelatinase-associated lipocalin regulates epithelial morphogenesis in vitro. J Biol Chem 280: 7875-7882, 2005.

33. Cowland JB, Sorensen OE, Sehested M, et al: Neutrophil gelatinase-associated lipocalin is up-regulated in human epithelial cells by IL-1 $\beta$, but not by TNF- $\alpha$. J Immunol 171: 6630-6639, 2003.

34. Yang J, Goetz D, Li JY, et al: An iron delivery pathway mediated by a lipocalin. Mol Cell 10: 1045-1056, 2002.

35. Stockand JD and Sansom SC: Glomerular mesangial cells: electrophysiology and regulation of contraction. Physiol Rev 78: 723-744, 1998.

36. Schena FP, Strippoli GF and Wankelmuth P: Renal growth factors: past, present and future. Am J Nephrol 19: 308-312, 1999. 